\title{
Adaptive Sliding Mode Observation in a Network of Dynamical Systems
}

\author{
Prathyush P Menon, Christopher Edwards and Yuri B Shtessel \\ College of Engineering, Mathematics and Physical Science, University of Exeter, Exeter, EX4 4JN, UK; (E-mail: \\ P.M.Prathyush@exeter.ac.uk, C.Edwards@exeter.ac.uk) \\ Department of Electrical Engineering, University of Alabama, Huntsville, Alabama, USA; (E-mail: \\ shtessel@ece.uah.edu)
}

\begin{abstract}
SUMMARY
This paper considers the problem of reconstructing state information in all the nodes of a complex network of dynamical systems. The individual nodes comprise a known linear part and unknown but bounded uncertainties in certain channels of the system. A supervisory adaptive sliding mode observer configuration is proposed for estimating the states. A linear matrix inequality (LMI) approach is suggested to synthesise the gains of the sliding mode observer. Although deployed centrally to estimate all the states of the complex network, the design process depends only on the dynamics of an individual node of the network. The methodology is demonstrated by considering a network of Chua oscillators.
\end{abstract}

KEY WORDS: Sliding modes, Observation, Adaptation

\section{INTRODUCTION}

The study of networks comprising a large number of dynamical systems, has been the subject of significant research in recent years [27, 18, 11,39]. Centralised monitoring of the overall system behaviour is important, and particulary for real engineering systems, safety and reliability is an important issue. In this paper, a specific representation of the general scale-free dynamical network model, as reported in [26], and compatible with the one studied in [39], will be adopted. The motivation for the work reported in this paper stems from the requirements of increasing the level of autonomy in 'systems of systems': i.e. a collection of dynamical systems operated over a network, and controlled to perform synchronously (or coordinated to attain a common objective). These problems are prevalent in many areas of research - for example mobile robots, formation flying of UAV's and satellites, vehicle platoons, and distributed state estimation applications such as localisation. For further details, see [27].

State observers for large scale systems have been employed in a number of different contexts. In [16], for a network of agents modelled by second order dynamics (position and velocity), the states of the neighbourhood are estimated (at agent level), and these estimates are then used in a dynamic coordination law to ensure leader following. Observers to estimate the states of the neighbourhood agents (posed as the dual problem of controller design) have been reported in [43]. Refs. [16, 43] employ full order state observers, whilst reduced order functional observers are proposed in [17] for finite dimensional networks of linear systems. In [17], the parallel Luenberger observers are designed for each node, with an identical neighbourhood interconnection structure as that of the original network, and then observer based control laws have been devised to achieve consensus.

${ }^{*}$ Correspondence to: College of Engineering, Mathematics and Physical Science, University of Exeter, Exeter, EX4 4JN, U.K. (e-mail: P.M.Prathyush@exeter.ac.uk, C.Edwards@exeter.ac.uk)

Department of Electrical Engineering, University of Alabama, Huntsville, Alabama, USA; (E-mail: shtessel@ece.uah.edu) 
In [23] the leader-follower consensus problem is addressed using functional observers to estimate the states of neighbourhood agents from only the output information of the neighbourhood agents (where the dynamics of the agents is assumed to be linear). A cooperative fault accommodation framework is proposed in [3] which makes use of relative state measurements, and is extended to the general case of formation flying of vehicles in a planetary orbital environment with absolute measurements in [4]. Large scale observer based schemes have also been applied to power networks to detect conventional electrical faults [1], but also cyber physical attacks [33]. The work in this paper addresses a similar observation problem, extended to a certain class of nonlinear/uncertain systems, based on sliding mode concepts.

Sliding mode observers, which constitute a specific subclass of robust nonlinear observers, have been studied extensively $[7,22,12,8,9,5,14,13,6,15,30]$. The underlying idea is to drive the state estimation error onto a sliding surface in finite time and then achieve at least asymptotic and often finite time convergence of the state estimate to the real state. In the early paper [2], an adaptive sliding mode observer was designed to synchronise a master-slave configuration for a class of nonlinear systems. In [31], a sliding mode observer was used for cryptography based on chaotic masking modulation, which is another relevant area of synchronisation involving coupled masterslave nonlinear systems. Furthermore, adaptive sliding mode control has also been applied in [41] for synchronisation of general master slave systems. Monitoring of complex networks from a fault detection perspective have also become prevalent. Patton and co-workers have proposed a generic framework for FDI in networked systems [29, 19] based on unknown input observers, whilst [28, 24] have considered fault detection problems in networks based on relative measurement information. These early papers focused on generic problem formulations but more recently, problem specific contributions have appeared: for example in [40], a bespoke sliding mode observer has been designed for online monitoring and fault detection in satellite formations. Disturbance estimates from a super-twist observer have also been exploited in [25] to enhance the robustness of formation control of satellites.

The focus of this paper is the synthesis of a centralised observer for a class of dynamical systems operating over an undirected network. The aim of this paper is to design, synthesise gains and analyse the convergence of the overall network system at an individual node level (i.e., independent of the number of nodes comprising the large scale system). The resulting design is a centralised monitoring scheme for the entire network, which is original in the context of the class of observers which are considered. The individual node level dynamics comprises a linear system and an unknown bounded nonlinear/uncertain part. The objective is to estimate the state of the network at a supervisory level from only measurement signals, using an adaptive sliding mode observer. The adaptive observer design for the network will be formulated as Linear Matrix Inequalities, which are solved at an individual node level, which is a crucial benefit of this approach.

\section{SYSTEM DESCRIPTION}

This paper considers a scale free network of $N$ interconnected dynamical systems, indexed as $1,2, \ldots, N$. The interconnections are assumed to be of bidirectional nature, and hence the individual sub-systems can be viewed as nodes in an undirected graph. Specifically the dynamics of the $i^{\text {th }}$ node is given by

$$
\begin{aligned}
& \dot{x}_{i}=A x_{i}+D \xi_{i}\left(x_{i}, u_{i}\right)+B u_{i}-\alpha(t) \sum_{j=1}^{N} \mathcal{L}_{i j} \Gamma x_{j} \\
& y_{i}=C x_{i}
\end{aligned}
$$

where $x_{i} \in \mathbb{R}^{n}, u_{i} \in \mathbb{R}^{m}$ and $y_{i} \in \mathbb{R}^{p}$ represent the state, the control inputs and the measured outputs respectively. The terms $\xi_{i}$ represent an unknown bounded uncertainty or a disturbance impacting on the dynamics. Although unknown, each $\xi_{i}$ term is assumed to be bounded. Specifically, it is assumed $\left\|\xi_{i}\left(x_{i}, u_{i}\right)\right\| \leq \beta_{0}$, where the constant term $\beta_{0}$ is assumed to be unknown but finite. 


\section{Assumption 1}

The triple $(A, D, C)$ consists of constant matrices $A \in \mathbb{R}^{n \times n}, D \in \mathbb{R}^{n \times q}$ and $C \in \mathbb{R}^{p \times n}$, where $C$ and $D$ are full row and column rank respectively, and $p \geq q$.

\section{Assumption 2}

The system $(A, D, C)$ is minimum phase.

\section{Assumption 3}

The distribution matrices satisfy the condition, $\operatorname{rank}(C D)=q$.

In (1), the matrix $\Gamma=\tau_{i j} \in \mathbb{R}^{n \times n}$ captures the local coupling configuration among the states of the nodes. It is assumed that entries of $\Gamma$ are either 0 or 1 and represent the existence or nonexistence of coupling in the respective channels of the network. The Laplacian of the underlying graph $\mathcal{G}$, and written as $\mathcal{L} \in \mathbb{R}^{N \times N}$, captures the interconnections of the network. Whenever node $i$ interacts with node $j$, there exists an edge between nodes $i$ and $j$, and $\mathcal{L}_{i j}=\mathcal{L}_{j i}=-1$, otherwise $\mathcal{L}_{i j}=\mathcal{L}_{j i}=0$. The diagonal terms are given by $\mathcal{L}_{i i}=k_{i}$, for $i=1, \ldots, N$ where $k_{i}$ is the degree of node $i$, and represents the total number of connections incident at the $i^{t h}$ node. The resulting graph Laplacian is a symmetric positive semi-definite matrix. Furthermore, the smallest eigenvalue of $\mathcal{L}$ is zero and the corresponding eigenvector is given by $\mathbf{1}$ [32]. This "row sum equal to zero" property will be exploited in this paper. The scalar $\alpha(t) \in \mathbb{R}^{+}$for all $t \in \mathbb{R}^{+}$is assumed to be a known time varying coupling strength, with a known upper bound $\hat{\alpha}=\sup _{t \in \mathbb{R}^{+}} \alpha(t)$.

The problem addressed in this paper is to design an adaptive sliding mode observer, at a supervisory level, to robustly reconstruct the states of the entire network $x=\operatorname{Col}\left(x_{1}, \ldots, x_{N}\right)$ based only on information about the measured outputs $y=\operatorname{Col}\left(y_{1}, \ldots, y_{N}\right)$ and the control inputs $u=\operatorname{Col}\left(u_{1}, \ldots, u_{N}\right)$.

\section{SLIDING MODE NETWORK OBSERVER}

Exploiting Assumptions 2 and 3, there exists a non-singular transformation $x_{i} \mapsto T_{0} x_{i}$ for each node, which transforms the original co-ordinates into ones in which the triple $(A, D, C)$ possesses the following canonical form(for example see Section 5.3 in [9])

$$
A=\left[\begin{array}{ll}
A_{11} & A_{12} \\
A_{21} & A_{22}
\end{array}\right] \quad D=\left[\begin{array}{c}
0 \\
D_{0}
\end{array}\right] \quad C=\left[\begin{array}{ll}
0 & C_{2}
\end{array}\right]
$$

where $A_{11} \in \mathbb{R}^{(n-p) \times(n-p)}, D_{0} \in \mathbb{R}^{q \times q}$ is non-singular, and $C_{2} \in \mathbb{R}^{p \times p}$ is orthogonal. Define $A_{211}$ as the top $p-q$ rows of $A_{21}$, then it can be shown the pair $\left(A_{11}, A_{211}\right)$ is detectable and the unobservable modes of $\left(A_{11}, A_{211}\right)$ are the invariant zeros of $(A, D, C)$ [10]. For streamlining the notation used later in the paper, define $D_{2}=\mathcal{C}$ ol $\left[\begin{array}{cc}0 & D_{0}\end{array}\right] \in \mathbb{R}^{p \times q}$ as the bottom $p$ rows of $D$. This coordinate system will be used for the observer synthesis.

In the coordinate system associated with the canonical form in (3), write the configuration matrix (which has no particular structure) as

$$
\Gamma=\left[\begin{array}{ll}
\Gamma_{11} & \Gamma_{12} \\
\Gamma_{21} & \Gamma_{22}
\end{array}\right]
$$

where $\Gamma_{22} \in \mathbb{R}^{p \times p}$.

Let $z_{i} \in \mathbb{R}^{n}$ be the state estimate of the $i^{t h}$ node which satisfies

$$
\dot{z}_{i}=A z_{i}+B u_{i}-\alpha(t) \sum_{j=1}^{N} \mathcal{L}_{i j} \Gamma z_{j}-G_{l_{i}} e_{y_{i}}-G_{n_{i}} \nu_{i}, \quad i=1, \ldots, N
$$

where $e_{y_{i}}=C\left(x_{i}-z_{i}\right)$. Let the global output error estimate be given by $e_{y}=\operatorname{Col}\left(e_{y_{1}}, \ldots e_{y_{N}}\right)$. For use later in the paper, consistent with (3), create the partition $z_{i}=\operatorname{Col}\left(z_{1 i}, z_{2 i}\right)$ such that $z_{1 i} \in \mathbb{R}^{n-p}$ 
and $z_{2 i} \in \mathbb{R}^{p}$. The discontinuous injection terms $\nu_{i}$ in (5) are given by:

$$
\nu_{i}=-\rho(t) \frac{e_{y_{i}}}{\left\|e_{y_{i}}\right\|}, \quad \text { if } e_{y_{i}} \neq 0
$$

In (6) the (scalar) modulation function $\rho: \mathbb{R}_{+} \mapsto \mathbb{R}_{+}$, will be subject to an adaptive law, which will be defined later.

The design freedom in (5) is represented by the gain matrices $G_{l_{i}} \in \mathbb{R}^{n \times p}$ and $G_{n_{i}} \in \mathbb{R}^{n \times p}$. These must be selected to ensure the state estimation error is driven to the sliding surface in finite time. A suitable choice for the matrix $G_{n_{i}}$ in the co-ordinate system in (3) is $G_{n_{i}}=\operatorname{Col}\left(L C_{2}^{\mathrm{T}}, C_{2}^{\mathrm{T}}\right)$, where $L=\left[\begin{array}{ll}L^{o} & 0\end{array}\right]$ with $L^{o} \in \mathbb{R}^{(n-p) \times(p-q)}$ [10]. The matrices $L^{o}$ and $G_{l_{i}}$ will be determined later in the paper using an LMI optimisation procedure.

Define $e_{i}:=x_{i}-z_{i}$ as the state estimation error in the $i^{t h}$ node. It can be verified from (1) and (5) that in the coordinates of (3)

$$
\dot{e}_{i}=A e_{i}+D \xi_{i}\left(x_{i}, u_{i}\right)-\alpha(t) \sum_{j=1}^{N} \mathcal{L}_{i j} \Gamma e_{j}+G_{l_{i}} e_{y_{i}}+G_{n_{i}} \nu_{i}
$$

for $i=1, \ldots, N$. In the coordinates associated with (3), let $e_{1_{i}} \in \mathbb{R}^{n-p}$ and $e_{2_{i}} \in \mathbb{R}^{p}$ such that $\operatorname{Col}\left(e_{1_{i}}, e_{2_{i}}\right)=e_{i}$. Note that $e_{2_{i}}$ is directly related to the output error estimate $e_{y_{i}}$, since $e_{y_{i}}=$ $C_{2} e_{2}$ where $\operatorname{det}\left(C_{2}\right) \neq 0$ ( and in fact $C_{2}^{\mathrm{T}} C_{2}=C_{2} C_{2}^{\mathrm{T}}=I$, since by construction $C_{2}$ from (3) is orthogonal). For the error system in (7), the objective is to induce a sliding motion on

$$
\mathcal{S}=\bigcup_{i=1 \ldots N}\left(\mathcal{S}_{i}=\left\{\left(e_{1_{i}}, e_{2_{i}}\right) \in \mathbb{R}^{n} \mid e_{2_{i}}=0\right\}\right) .
$$

For each node, consider a change of coordinates from [42] of the form, $e_{i} \mapsto T_{L} e_{i}$, where

$$
T_{L}:=\left[\begin{array}{cc}
I_{n-p} & -L \\
0 & I_{p}
\end{array}\right]
$$

In the new coordinates, the plant matrix $A$ in (3) is written as

$$
\left[\begin{array}{ll}
\tilde{A}_{11} & \tilde{A}_{12} \\
A_{21} & \tilde{A}_{22}
\end{array}\right]=T_{L} A T_{L}^{-1}
$$

where $\tilde{A}_{11}:=\left(A_{11}-L^{o} A_{211}\right) \in \mathbb{R}^{(n-p) \times(n-p)}$ and the local coupling matrix terms in (4) are

$$
\left[\begin{array}{ll}
\tilde{\Gamma}_{11} & \tilde{\Gamma}_{12} \\
\Gamma_{21} & \tilde{\Gamma}_{22}
\end{array}\right]=T_{L} \Gamma T_{L}^{-1}
$$

where $\tilde{\Gamma}_{11}=\Gamma_{11}-L \Gamma_{21} \in \mathbb{R}^{(n-p) \times(n-p)}$. Here, it is assumed that the gain $L$ (or in fact $L^{o}$ ) can be chosen so that there exists a symmetric positive definite matrix $P_{1} \in \mathbb{R}^{(n-p) \times(n-p)}$ such that

$$
\begin{aligned}
P_{1} & <0 \\
-P_{1} \tilde{\Gamma}_{11}-\tilde{\Gamma}_{11}^{\mathrm{T}} P_{1} & \leq 0 \\
P_{1} \tilde{A}_{11}+\tilde{A}_{11}^{\mathrm{T}} P_{1}+\tilde{q} I_{n-p} & \leq 0
\end{aligned}
$$

where $\tilde{q} \in \mathbb{R}^{+}$is a chosen design scalar. The inequalities in (12)-(14) can be formulated as a convex optimization problem via a system of LMI's. In the LMI representation, the matrix variables $P_{1}$ and $M:=P_{1} L$ are used as decision variables. It can be seen from (12)-(14) that the LMI's to be solved are independent of the number of nodes in the network $(N)$ and only depend on $(n-p) \times(n-p)+(n-p) \times(p-q)$ decision variables. 


\section{Remark 1}

Suppose the system in (1)-(2) is treated as a centralised system

$$
\begin{aligned}
\dot{x} & =\mathbb{A}(t) x+\mathbb{B} u+\mathbb{F} \Xi \\
y & =\mathbb{C} x
\end{aligned}
$$

where $x \in \mathbb{R}^{N n}, u \in \mathbb{R}^{N m}, y \in \mathbb{R}^{N p}$ and $\Xi=\mathcal{C o l}\left(\xi_{1}, \ldots, \xi_{N}\right)$. In equations (15)-(16), the matrices are $\mathbb{A}(t)=\left(I_{N} \otimes A\right)-\alpha(t)(\mathcal{L} \otimes \Gamma), \mathbb{B}=\left(I_{N} \otimes B\right), \mathbb{C}=\left(I_{N} \otimes C\right)$, and $D=\left(I_{N} \otimes D\right)$, and in particular the specific structures of $(\mathbb{A}(t), \mathbb{D}, \mathbb{C})$ are ignored. Then it would be theoretically possible to design a centralised observer of the types proposed in $[35,36,38]^{\dagger}$. In such a design there would be $2 N n \times N p$ elements in the gain matrices to select, and quadratic Lyapunov function matrices with $(n N+1) N n / 2$ elements. In this paper all the computation depend only on the size of the individual node dynamics $n, p$, and $m$ and not on the size of the network. For large scale systems (say for $N$ of order 1000), from a purely computational view point the calculations will become unmanageable. For example it is highly likely the LMI based methods in [36] and [35] would become impractical. Using the symbolic approach in [38] is also likely to be infeasible. (In fact it is highly probable even traditional robust pole placement algorithms [20] will be found wanting from a numerical perspective.

Consistent with (3), partition the error state vector in the transformed coordinates associated with (9) as $\operatorname{Col}\left(\tilde{e}_{1_{i}}, e_{2_{i}}\right)=T_{L} e_{i}$, for $i=1, \ldots, N$ where by definition $\tilde{e}_{1_{i}}:=e_{1_{i}}-L e_{2_{i}}$. In the partitioned form, define the observer gain matrix $G_{l_{i}}=\operatorname{Col}\left(G_{1_{i}}, G_{2_{i}}\right)$, for $i=1, \ldots, N$ where $G_{1_{i}} \in \mathbb{R}^{(n-p) \times p}$. In the coordinates of (9), the error system from (7) can be written as

$$
\begin{aligned}
& \dot{\tilde{e}}_{1_{i}}=\tilde{A}_{11} \tilde{e}_{1_{i}}+\tilde{A}_{12} e_{2_{i}}+\left[I_{n-p}-L\right] G_{l_{i}} e_{y_{i}}-\alpha(t) \sum_{j=1}^{N} \mathcal{L}_{i j}\left(\tilde{\Gamma}_{11} \tilde{e}_{1_{j}}+\tilde{\Gamma}_{12} e_{2_{j}}\right) \\
& \dot{e}_{2_{i}}=A_{21} \tilde{e}_{1_{i}}+\tilde{A}_{22} e_{2_{i}}+D_{2} \xi_{i}(.)+G_{2_{i}} e_{y_{i}}+C_{2}^{\mathrm{T}} \nu-\alpha(t) \sum_{j=1}^{N} \mathcal{L}_{i j}\left(\Gamma_{21} \tilde{e}_{1_{j}}+\tilde{\Gamma}_{22} e_{2_{j}}\right)
\end{aligned}
$$

for $i=1, \ldots, N$. Now define explicitly

$$
\begin{aligned}
G_{2_{i}} & :=\Psi C_{2}^{\mathrm{T}}-\tilde{A}_{22} C_{2}^{\mathrm{T}} \\
G_{1_{i}} & :=L G_{2_{i}}-\tilde{A}_{12} C_{2}^{\mathrm{T}}
\end{aligned}
$$

for $i=1, \ldots, N$, where $\Psi \in \mathbb{R}^{p \times p}$ is a designer selected Hurwitz matrix.

Let $\tilde{e}_{1}=\operatorname{Col}\left(\tilde{e}_{1_{1}}, \ldots \tilde{e}_{1_{N}}\right)$ and $e_{2}=\operatorname{Col}\left(e_{2_{1}}, \ldots e_{2_{N}}\right)$ then substituting $G_{1_{i}}$ and $G_{2_{i}}$ defined above into (17)-(18), the error dynamics in the transformed coordinates can be written as

$$
\begin{aligned}
\dot{\tilde{e}}_{1}= & \left(\left(I_{N} \otimes \tilde{A}_{11}\right)-\alpha(t)\left(\mathcal{L} \otimes \tilde{\Gamma}_{11}\right)\right) \tilde{e}_{1}+\Phi_{1}\left(t, e_{2}\right) \\
\dot{e}_{2}= & \left(\left(I_{N} \otimes A_{21}\right)-\alpha(t)\left(\mathcal{L} \otimes \Gamma_{21}\right)\right) \tilde{e}_{1}+\Phi_{2}\left(t, e_{2}\right) \\
& +\left(I_{N} \otimes \Psi\right) e_{2}+\left(I_{N} \otimes D\right) \xi(x, u)+\left(I_{N} \otimes C_{2}^{\mathrm{T}}\right) \nu
\end{aligned}
$$

where $\nu:=\operatorname{Col}\left(\nu_{1}, \ldots \nu_{N}\right)$ and $\xi(x, u)=\operatorname{Col}\left(\xi_{1}(\cdot), \ldots, \xi_{N}(\cdot)\right)$. In the error systems (21) - (22), the injection terms $\Phi_{1}\left(t, e_{2}\right):=-\alpha(t)\left(\mathcal{L} \otimes \tilde{\Gamma}_{12}\right) e_{2}$ and $\Phi_{2}\left(t, e_{2}\right):=-\alpha(t)\left(\mathcal{L} \otimes \tilde{\Gamma}_{22}\right) e_{2}$. Since $\Phi_{1}\left(t, e_{2}\right)$ is linear with respect to $e_{2}$, it follows

$$
\left\|\Phi_{1}\left(t, e_{2}\right)\right\| \leq \alpha(t) K_{1}\left\|e_{2}\right\|
$$

for all $\left(t, e_{2}\right) \in \mathbb{R} \times \mathbb{R}^{p N}$, where $K_{1}=\left\|\left(\mathcal{L} \otimes \tilde{\Gamma}_{12}\right)\right\|$ is a positive scalar. As a precursor to analysing (21)-(22), consider the unforced time varying linear system

$$
\dot{\tilde{e}}_{1}=\left(I_{N} \otimes \tilde{A}_{11}-\alpha(t)\left(\mathcal{L} \otimes \tilde{\Gamma}_{11}\right)\right) \tilde{e}_{1}
$$

\footnotetext{
${ }^{\dagger}$ Technically the observers in $[35,36,38]$ are formulated for LTI systems, but if $\alpha(t)$ is known, the time varying system matrix $\mathbb{A}(t)$ could be written polytopically and the approaches in $[35,36]$ adapted accordingly.
} 
which can be thought of as the reduced order motion, if sliding occurs on $\mathcal{S}$. Consider a candidate Lyapunov function for (24) of the form $\mathcal{V}\left(\tilde{e}_{1}\right):=\tilde{e}_{1} \tilde{P} \tilde{e}_{1}$, where $\tilde{P}=\left(I_{N} \otimes P_{1}\right)$ and $P_{1}$ is the s.p.d matrix satisfying the conditions defined earlier in (13)-(14). Since the Laplacian matrix $\mathcal{L}$ is symmetric, by spectral decomposition, it can be written as

$$
\mathcal{L}=V \Lambda V^{\mathrm{T}}
$$

where $\Lambda=\operatorname{Diag}\left(\lambda_{1}, \ldots, \lambda_{N}\right)$ and the $\lambda_{i} \in \mathbb{R}^{+}$are the real non negative eigenvalues [32], and $V$ is an orthogonal matrix composed of the associated eigenvectors. As in [26], consider a change of coordinates $\tilde{e}_{1} \mapsto T_{v} \tilde{e}_{1}=\eta$ where

$$
T_{v}:=\left(V^{\mathrm{T}} \otimes I_{(n-p)}\right)
$$

Because $V^{\mathrm{T}} V=I_{N}, T_{v}$ in (26) is also an orthogonal transformation. Then from the properties of Kronecker products, $T_{v}\left(I_{N} \otimes \tilde{A}_{11}\right) T_{v}^{\mathrm{T}}=\left(I_{N} \otimes \tilde{A}_{11}\right)$ and

$$
T_{v}\left(\mathcal{L} \otimes \tilde{\Gamma}_{11}\right) T_{v}^{\mathrm{T}}=\left(V^{\mathrm{T}} \mathcal{L} V\right) \otimes \tilde{\Gamma}_{11}=\Lambda \otimes \tilde{\Gamma}_{11}
$$

Applying the transformation in (26) to equation (24), and exploiting the results identified above, it follows that

$$
\dot{\eta}=\left(I_{N} \otimes \tilde{A}_{11}-\alpha(t)\left(\Lambda \otimes \tilde{\Gamma}_{11}\right)\right) \eta
$$

Note that since $\Lambda$ is diagonal, (27) can be written in a node level representation as

$$
\dot{\eta}_{i}=\underbrace{\left(A_{11}-L A_{21}-\alpha(t) \lambda_{i} \tilde{\Gamma}_{11}\right)}_{\tilde{A}_{i}(t)} \eta_{i}
$$

for $\eta_{i}, i=1 \ldots N$ where $\eta=\operatorname{Col}\left(\eta_{1}, \ldots \eta_{N}\right)$. In the new coordinates $\mathcal{V}\left(\tilde{e}_{1}\right)$ becomes

$$
\mathcal{V}(\eta):=\eta^{\mathrm{T}} \tilde{P} \eta=\sum_{i=1}^{n} \eta_{i}^{\mathrm{T}} P_{1} \eta_{i}
$$

because $T_{v}$ is orthogonal and has the specific structure in (26). For the differential equations in (28) consider a candidate Lyapunov function $\mathcal{V}_{i}\left(\eta_{i}\right)=\eta_{i}^{\mathrm{T}} P_{1} \eta_{i}$, where $P_{1} \in \mathbb{R}^{(n-p) \times(n-p)}$ is the symmetric positive definite matrix obtained by solving (12)-(14). The time derivative of $\mathcal{V}_{i}\left(\eta_{i}\right)$ along the trajectories of (28) is given by

$$
\begin{aligned}
\left.\dot{\mathcal{V}}_{i}\left(\eta_{i}\right)\right|_{(28)} & =\eta_{i}^{\mathrm{T}}\left(P_{1} \tilde{A}_{i}(t)+\tilde{A}_{i}(t)^{\mathrm{T}} P_{1}\right) \eta_{i} \\
& =\eta_{i}^{\mathrm{T}}\left(P_{1}\left(A_{11}-L A_{21}\right)+\left(A_{11}-L A_{21}\right)^{\mathrm{T}} P_{1}\right) \eta_{i}-\alpha(t) \lambda_{i} \eta_{i}^{\mathrm{T}}\left(P_{1} \tilde{\Gamma}_{11}+\tilde{\Gamma}_{11}^{\mathrm{T}} P_{1}\right) \eta_{i}
\end{aligned}
$$

Since $\alpha(t)$ is a positive bounded scalar and $\lambda_{i} \geq 0, \alpha(t) \lambda_{i} \geq 0$. Then from (13) $\left(P_{1} \tilde{\Gamma}_{11}+\tilde{\Gamma}_{11}^{\mathrm{T}} P_{1}\right)$ is positive semi-definite, and the time-varying expression $-\alpha(t) \lambda_{i} \eta_{i}^{\mathrm{T}}\left(P_{1} \tilde{\Gamma}_{11}+\widetilde{\Gamma}_{11}^{\mathrm{T}} P_{1}\right) \eta_{i}$ in (29) is negative semi-definite for all $\eta_{i} \neq 0$ and $\dot{\mathcal{V}}_{i}\left(\eta_{i}\right) \leq-\tilde{q}\left\|\eta_{i}\right\|^{2}$, where $\tilde{q}$ is the positive scalar quantity in (14). Consequently $\dot{\mathcal{V}}\left(\tilde{e}_{1}\right) \leq-\tilde{q}\left\|\tilde{e}_{1}\right\|^{2}$ in the case when $\Phi_{1}\left(t, e_{2}\right)=0$ in (21).

Further define $\tilde{\mathcal{V}}=\sqrt{\mathcal{V}}$. Since $\mathcal{V}$ is positive definite, this is well defined. With these definitions, and using Rayleigh's inequality [21]

$$
\lambda_{\min }\left(P_{1}\right)\left\|e_{1}\right\|^{2} \leq \mathcal{V}\left(\tilde{e}_{1}\right) \leq \lambda_{\max }\left(P_{1}\right)\left\|e_{1}\right\|^{2},
$$

it follows that the derivative of $\mathcal{V}$ along the trajectories of (21) is

$$
\left.\dot{\mathcal{V}}\right|_{(21)} \leq-\frac{\tilde{q}}{\lambda_{\max }\left(P_{1}\right)} \tilde{\mathcal{V}}^{2}+2 \mathcal{V} \sqrt{\lambda_{\max }\left(P_{1}\right)} \alpha(t) K_{1}\left\|e_{2}\right\|
$$

Since by definition $\mathcal{V}=\tilde{\mathcal{V}}^{2}$ it follows $\dot{\mathcal{V}}=2 \dot{\tilde{\mathcal{V}}} \tilde{\mathcal{V}}$ and hence using (30) yields $\dot{\tilde{\mathcal{V}}}\left(\tilde{e}_{1}\right)$ as

$$
\dot{\tilde{\mathcal{V}}}\left(\tilde{e}_{1}\right) \leq-\frac{\tilde{q}}{2 \lambda_{\max }\left(P_{1}\right)} \tilde{\mathcal{V}}+\alpha(t) \sqrt{\lambda_{\max }\left(P_{1}\right)} K_{1}\left\|e_{2}\right\|
$$


For notational simplicity the inequality in (31) can be written as

$$
\dot{\tilde{\mathcal{V}}} \leq-m_{0} \tilde{\mathcal{V}}+m_{1} \alpha(t)\left\|e_{2}(t)\right\|
$$

for appropriate known positive scalars $m_{0}$ and $m_{1}$ and a known coupling strength $\alpha(t)$.

Define adaptive filter dynamics as

$$
\dot{r}_{1}(t)=-m_{0} r_{1}(t)+m_{1} \alpha(t)\left\|e_{2}(t)\right\|
$$

where $r_{1} \in \mathbb{R}$ represents the adapting scalar element. Note that $e_{y_{i}}=C_{2} e_{2_{i}}$, where $C_{2}$ is orthogonal, which implies $\left\|e_{y_{i}}\right\|=\left\|e_{2_{i}}\right\|$ and $\left\|e_{y}\right\|=\left\|e_{2}\right\|$. Hence (33) can be written as

$$
\dot{r}_{1}(t)=-m_{0} r_{1}(t)+m_{1} \alpha(t)\left\|e_{y}(t)\right\|
$$

From first principles, it can be seen $\left\|r_{1}(t)\right\|>\tilde{\mathcal{V}}(t)$ for all time $t>0$, provided $r_{1}(0) \geq \tilde{\mathcal{V}}(0)$. Note that $r_{1}(t)$ is an adaptive gain, which can be realized in real time, and depends only on $\left\|e_{y}\right\|$ which is known. Consequently if $r_{1}(0)>\tilde{\mathcal{V}}(0)$ then

$$
r_{1}(t)^{2}>\mathcal{V}(t) \geq \lambda_{\min }\left(P_{1}\right)\left\|\tilde{e}_{1}\right\|^{2} \Longrightarrow r_{1}(t) \geq \sqrt{\lambda_{\min }\left(P_{1}\right)}\left\|\tilde{e}_{1}(t)\right\|
$$

for all time. Now consider the dynamics in (22). Again by linearity it can be argued that

$$
\left\|\Phi_{2}\left(t, e_{2}\right)\right\| \leq \alpha(t) K_{2}\left\|e_{2}\right\|
$$

where $K_{2}=\left\|\left(\mathcal{L} \otimes \tilde{\Gamma}_{22}\right)\right\|$ is some positive constant.

Define a second adaptive scalar gain $r_{2}$ according to the following dynamics

$$
\dot{r}_{2}(t)=\gamma\left\|e_{y}(t)\right\|
$$

where $\gamma$ is a positive design scalar. The objective is now to show the standard sliding mode reachability condition $[9,37]$ can be satisfied to show that a sliding motion occurs in finite time. The choice is such that $r_{2}(t)$ remains bounded and $e_{2}(t)$ asymptotically becomes zero. Define scalar error variable as

$$
e_{r}(t):=\left\|D_{2}\right\| \beta_{0}-r_{2}(t)
$$

Clearly if $e_{r}(t)$ can be shown to be bounded, then $r_{2}(t)$ is bounded and the asymptotic convergence of $e_{2}(t)$ is true. In what follows, it is advantageous to assume that the matrix $\Psi$ in (19) has the special form $\Psi:=-\psi I_{p}$ where $\psi \in \mathbb{R}^{+}$is a design scalar. The objective is now to show $e_{2}(t) \rightarrow 0$. To this end consider the function

$$
\mathbf{V}=e_{2}^{\mathrm{T}} e_{2}+\frac{1}{\gamma} e_{r}^{2}
$$

Taking the derivative of $\mathbf{V}$ in (38) along the trajectory of (22) and the adaptation dynamics defined in (36), yields

$$
\begin{aligned}
\dot{\mathbf{V}} \leq & \frac{2}{\gamma} e_{r} \dot{e}_{r}+2\left\|e_{2}\right\|\left\|\left(I_{N} \otimes A_{21}\right)\right\|\left\|\tilde{e}_{1}\right\|-2 \psi\left\|e_{2}\right\|^{2} \\
& +2 \alpha(t)\left\|\left(\mathcal{L} \otimes \Gamma_{21}\right)\right\|\left\|e_{2}\right\|\left\|\tilde{e}_{1}\right\|+2 \alpha(t)\left\|\left(\mathcal{L} \otimes \tilde{\Gamma}_{22}\right)\right\|\left\|e_{2}\right\|^{2} \\
& +2 K_{2}\left\|e_{2}\right\|^{2}+2\left\|e_{2}\right\|\left\|D_{2}\right\| \beta_{0}-2 e_{2}^{\mathrm{T}}\left(I_{N} \otimes C_{2}^{\mathrm{T}}\right) \nu
\end{aligned}
$$

It is easy to see from the properties of Kronecker products that

$$
e_{2}^{\mathrm{T}}\left(I_{N} \otimes C_{2}^{\mathrm{T}}\right)=e_{2}^{\mathrm{T}}\left(I_{N} \otimes C_{2}\right)^{\mathrm{T}}=\left(\left(I_{N} \otimes C_{2}\right) e_{2}\right)^{\mathrm{T}}=e_{y}^{\mathrm{T}}
$$

And then from the definition of $\nu$ in (6), it follows:

$$
e_{2}^{\mathrm{T}}\left(I_{N} \otimes C_{2}^{\mathrm{T}}\right) \nu=-\rho \sum_{i=1}^{N}\left\|e_{y i}\right\| \leq-\rho\left\|e_{y}\right\|
$$


Consequently, if the design scalar $\psi$ is chosen to satisfy $\psi>\left(\alpha(t)\left\|\left(\mathcal{L} \otimes \tilde{\Gamma}_{22}\right)\right\|+K_{2}\right)$ then

$$
\begin{aligned}
\dot{\mathbf{V}} \leq & 2\left\|e_{2}\right\|\left\|\left(I_{N} \otimes A_{21}\right)\right\|\left\|\tilde{e}_{1}\right\|+2 \alpha(t)\left\|\left(\mathcal{L} \otimes \Gamma_{21}\right)\right\|\left\|e_{2}\right\|\left\|\tilde{e}_{1}\right\| \\
& +2\left\|e_{2}\right\|\left\|D_{2}\right\| \beta_{0}-2 \rho\left\|e_{y}\right\|+\frac{2}{\gamma} e_{r} \dot{e}_{r}
\end{aligned}
$$

Let $a_{21}:=\left\|I_{N} \otimes A_{21}\right\|=\left\|A_{21}\right\|$ and compute a bound $\gamma_{21} \geq \alpha(t)\left\|\mathcal{L} \otimes \Gamma_{21}\right\|$. (Since $\alpha(t)$ is bounded by $\hat{\alpha}$ then such a constant exists.) Moreover, note that $r_{1}(t)>\sqrt{\lambda_{\min }\left(P_{1}\right)}\left\|\tilde{e}_{1}(t)\right\|$ for all $t>0$. Using these norm bounds and the facts $\left\|e_{y}\right\|=\left\|e_{2}\right\|$ in (40), yields

$$
\dot{\mathbf{V}} \leq 2\left(\frac{a_{21}+\gamma_{21}}{\sqrt{\lambda_{\min }\left(P_{1}\right)}} r_{1}(t)+\left\|D_{2}\right\| \beta_{0}-\rho(t)\right)\left\|e_{2}\right\|+\frac{2}{\gamma} e_{r} \dot{e}_{r}
$$

Define the modulation gain $\rho(t)$ in $(6)$ as

$$
\rho(t):=\frac{\left(a_{21}+\gamma_{21}\right)}{\sqrt{\lambda_{\min }\left(P_{1}\right)}} r_{1}(t)+r_{2}(t)+r_{0}
$$

where $r_{0}$ is a positive design scalar. Substituting $\rho(t)$ in (41) yields

$$
\begin{aligned}
\dot{\mathbf{V}} & \leq 2\left\|D_{2}\right\| \beta_{0}\left\|e_{2}\right\|-2 r_{2}(t)\left\|e_{2}\right\|-2 r_{0}\left\|e_{2}\right\|+\frac{2}{\gamma} e_{r} \dot{e}_{r} \\
& =2\left\|e_{2}\right\|\left(\left\|D_{2}\right\| \beta_{0}-r_{2}(t)\right)-2 r_{0}\left\|e_{2}\right\|+\frac{2}{\gamma} e_{r} \dot{e}_{r}
\end{aligned}
$$

Using the definition of $e_{r}$ in (37),

$$
\dot{\mathbf{V}} \leq 2 e_{r}\left\|e_{2}\right\|-2 r_{0}\left\|e_{2}\right\|+\frac{2}{\gamma} e_{r} \dot{e}_{r}
$$

It follows from (37) that $\dot{e}_{r}=-\dot{r}_{2}$, then substituting for $\dot{r}_{2}$ from (36) yields $\dot{e}_{r}=-\gamma\left\|e_{2}\right\|$. Consequently from inequality (44) it is obvious that

$$
\dot{\mathbf{V}} \leq-2 r_{0}\left\|e_{2}\right\|
$$

Since $\dot{\mathbf{V}} \leq 0$ it follows that the signals $e_{2}$ and $e_{r}$ remain bounded and hence $r_{2}(t)$ remains bounded. Furthermore from (45), using Barbalat's lemma [21], $\left\|e_{2}(t)\right\| \rightarrow 0$ asymptotically.

In addition, if over an interval of time [ $\left.t_{1} \quad t_{2}\right]$ the gain $r_{2}(t)$ satisfies $r_{2}(t)>\left\|D_{2} \xi(t)\right\|$, then sliding will occur within $t_{s}=\left\|e_{2}\left(t_{1}\right)\right\| / r_{0}$ units of time provided $t_{1}+t_{s} \leq t_{2}$. This follows from employing the Lyapunov function $\mathcal{V}_{s}=e_{2}^{\mathrm{T}} e_{2}$ and noting that, using arguments similar to those leading up to (43), in this situation

$$
\begin{aligned}
\dot{\mathcal{V}}_{s} & \leq 2\left\|D_{2}\right\|\|\xi\|\left\|e_{2}\right\|-2 r_{2}(t)\left\|e_{2}\right\|-2 r_{0}\left\|e_{2}\right\| \\
& \leq-2 r_{0}\left\|e_{2}\right\|
\end{aligned}
$$

if $r_{2}(t)>\left\|D_{2} \xi(t)\right\|$. Consequently

$$
\dot{\mathcal{V}}_{s} \leq-2 r_{0} \sqrt{\mathcal{V}_{s}}
$$

which implied $\left\|e_{2}\right\| \equiv 0$ in a time $t_{s} \leq\left\|e_{2}\left(t_{1}\right)\right\| / r_{0}$. During sliding, $e_{2}=0$ and from (36) the gain $r_{2}(t)$ no longer increases. Sliding will be maintained provided the gain $r_{2}\left(t_{1}+t_{s}\right)>\left\|D_{2} \xi(t)\right\|$ for all $t>t_{1}+t_{s}$. If this does not hold (which is possible if $r_{2}\left(t_{1}+t_{s}\right)<\left\|D_{2}\right\| \beta_{0}$ ) then sliding may be temporarily broken, and then from (36), the gain $r_{2}(t)$ will increase and sliding will be regained in finite time using arguments similar to those above. If at any point $r_{2}(t)>\left\|D_{2}\right\| \beta_{0}$, sliding will be achieved and maintained for all subsequent time.

During a sliding mode in which $e_{y} \equiv 0$, it follows $e_{2}=0$ and the reduced order sliding mode is governed by (24) (or equivalently (27)). The system was shown to be quadratically stable using the Lyapunov function $\mathcal{V}\left(\tilde{e}_{1}\right)$, if $P_{1}$ satisfies (12)-(14). 


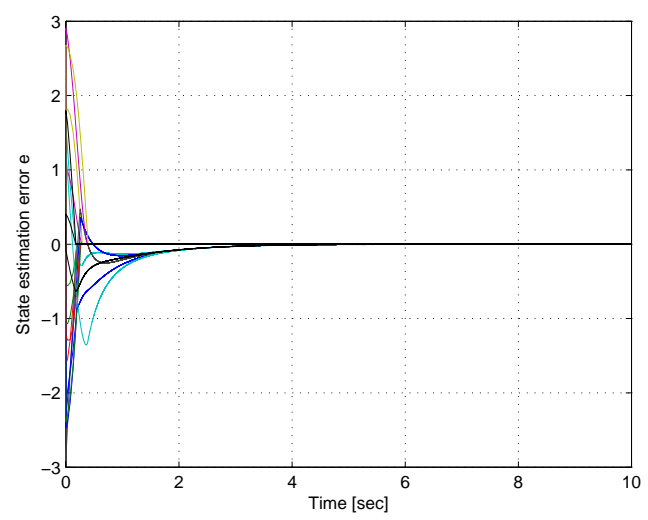

Figure 1. State estimation error

\section{NUMERICAL EXAMPLE}

To demonstrate the theory developed in this paper, a network of six Chua oscillators is considered, hence $N=6$. Here a Lur'e type ([21]) representation for Chua's circuit will be used, which ties in with the preceding development. Specifically here it is assumed that

$$
\begin{aligned}
& \dot{x}_{i}=A x_{i}+D \xi_{i}\left(x_{i}\right)-\alpha(t) \sum_{j=1}^{N} \mathcal{L}_{i j} \Gamma x_{j}+B u_{i} \\
& y_{i}=C x_{i}
\end{aligned}
$$

where

$$
A=\left[\begin{array}{ccc}
-a l_{1} & a & 0 \\
1 & -1 & 1 \\
0 & -b & 0
\end{array}\right], D=\left[\begin{array}{c}
-a\left(l_{0}-l_{1}\right) \\
0 \\
0
\end{array}\right], C=\left[\begin{array}{lll}
1 & 0 & 0 \\
0 & 1 & 0
\end{array}\right]
$$

The nonlinearity is given by $\xi_{i}\left(x_{i}\right)=\frac{1}{2}\left(\left|x_{i 1}+c\right|-\left|x_{i 1}-c\right|\right)$, which has a sector bound $[0,1]$. The chosen values of the parameters in (50) are $a=9, b=14.286, c=1, l_{0}=-1 / 7, l_{1}=2 / 7$ in order to obtain double scroll attractor behaviour [39, 34]. The underlying graph topology of the Chua oscillators, which are assumed to be coupled via resistors, is based on the nearest neighbour rule [27] which naturally defines $\mathcal{L}_{i j}$ in (48). The interconnection matrix $\Gamma=\operatorname{Diag}(1,0,0)$ models the interconnection among the states of the Chua oscillators. The time varying coupling strength $\alpha(t)$ is modelled as a sinusoidal signal with an input frequency of $0.5 \mathrm{~Hz}$, amplitude 0.5 and bias 1.0 . This ensures $\alpha(t) \geq 0$ and $\alpha(t)$ has a known upper bound $\hat{\alpha}=2$.

For simulation purposes, a controller is required to ensure synchronisation. As in [34], the control input matrix $B$ in (48) is taken as the identity and the state error feedback controller from [34] is exploited. Synchronisation to a pre-defined master behaviour (with initial conditions $\left.x_{\text {master }}(0)=[0.0196,0.6813,0.3795]\right)$ is obtained.

The main objective is robust reconstruction of all the node states in the network. It is easy to check the triple $(A, D, C)$ satisfies the Assumptions (1) - (3). The 4-block partition structure in (3) is then obtained by suitable coordinate transformation. The states of every node of the network are estimated from the measurement signals $y_{i}$ using the adaptive sliding mode observer in (5). Here, one assumption is that each node communicates its output information to the supervisory monitoring one in order to compute $\left\|e_{y}\right\|$ centrally, since this information is used in the adaptive filter dynamics in (34) and (36). For implementation purposes the expression in (36) is replaced by an adaptation law involving a 'dead-zone' so that $\dot{r}_{2}(t)=0$ if $\left\|e_{y}\right\| \leq \epsilon$ otherwise $\dot{r}_{2}(t)=\gamma\left\|e_{y}\right\|$ where $\epsilon$ is a small positive scalar. In the simulations which follow $\epsilon=0.0001$. The design freedom $\tilde{q} \in \mathbb{R}^{+}$in (14) has been chosen as unity. 

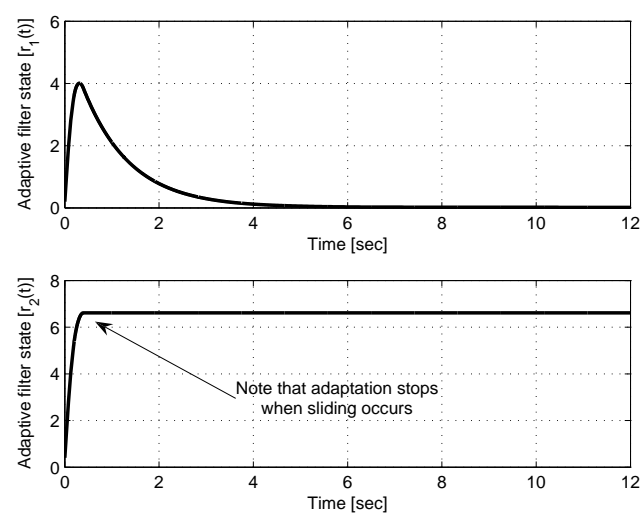

Figure 2. The states of the adaptive filter dynamics $\left(r_{1}, r_{2}\right)$

Solving the inequalities in (13)-(14), a Lyapunov matrix, $P_{1}$ is synthesized. Appropriate gains for the adaptive law in (34) and (36) have been selected as $m_{0}=-1, m_{1}=3.0425$ and $\gamma=5$. In (42), the bounds and the design scalars are selected as $a_{21}=1, \gamma_{21}=0, K_{1}=4.3028, K_{2}=0$ and $r_{0}=2$. Figure 1 shows the entire state estimation error $e=\operatorname{Col}\left(e_{1}, \ldots, e_{6}\right)$. Figure 2 shows the
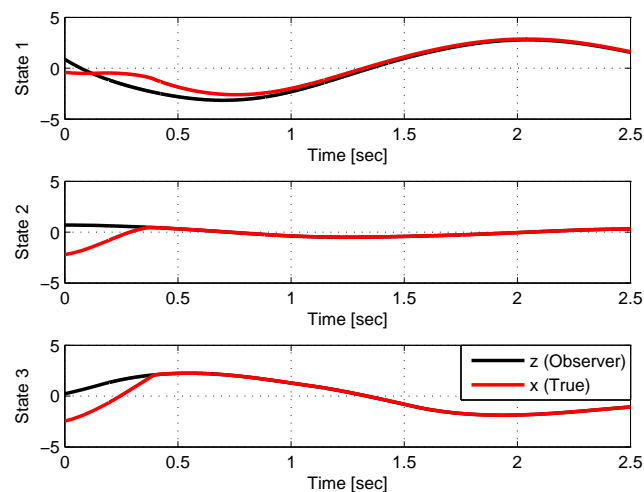

Figure 3. State estimation of one individual node

states $r_{1}(t)$ and $r_{2}(t)$ of the adaptive filter dynamics defined in (34) and (36) (shown only for a short period of time). In Figure 2, the adaptation of the gains stops at the time instant sliding occurs. To demonstrate the efficacy of the proposed scheme, an individual node has been selected randomly and the true states and the estimated states are shown in Figure 3.

\section{CONCLUSION}

This paper has proposed an adaptive sliding mode observer scheme for robustly reconstructing state information in a complex network of dynamical systems. The proposed centralised observer robustly reconstructs the states in the presence of a varying coupling strength of known bound, and a class of unknown bounded uncertainties/disturbances. The stability conditions derived for the network observer depend only on the dimensions of a typical individual node in the network. The design procedure to synthesize the observer gain is formulated as an LMI optimisation problem and performed at a node level. As such the number of decision variables is independent of the number of 
nodes in the network. A network of Chua circuits with six nodes is used to demonstrate the novelty of the proposed approach.

\section{REFERENCES}

1. M. Aldeen and F. Crusca. Observer-based fault detection and identification scheme for power systems. IEE Proceedings - Generation, Transmission and Distribution, 153:71-79, 2006.

2. A. Azemi and E. Yaz. Sliding mode adaptive observer approach to chaotic synchronization. Journal of Dynamic Systems, Measurement, and Control, 122:758-765, 2000.

3. S.M. Azizi and K. Khorasani. A hierarchical architecture for cooperative fault accommodation of formation flying satellites in deep space. American Control Conference, 2009.

4. S.M. Azizi and K. Khorasani. Cooperative actuator fault accommodation of formation flying vehicles with absolute measurements. In Proc. of $49^{\text {th }}$ IEEE Conference on Decision and Control, 2010.

5. F.J. Bejarano, L. Fridman, and A. Poznyak. Hierarchical observer for strongly detectable systems via second order sliding mode. In Proceedings of the IEEE Conference on Decision and Control, pages 3709-3713, 2007.

6. W. Chen and M. Saif. Actuator fault diagnosis for uncertain linear systems using a high-order sliding-mode robust differentiator. International Journal of Robust and Nonlinear Control, 18:413-426, 2007.

7. S. Drakunov and V.I. Utkin. Sliding mode observers: tutorial. In Proceedings of the 34th IEEE Conference of Decision and Control, pages 3376-3378, 1995.

8. C. Edwards and S.K. Spurgeon. On the development of discontinuous observers. International Journal of Control, 59:1211-1229, 1994.

9. C. Edwards and S.K. Spurgeon. Sliding Mode Control: Theory and Applications. Taylor \& Francis, 1998.

10. C. Edwards, S.K. Spurgeon, and R.J. Patton. Sliding mode observers for fault detection. Automatica, 36:541-553, 2000.

11. J. A. Fax and R. M. Murray. Information flow and cooperative control of vehicle formations. IEEE Trans. Aut. Control, 49:1465-1476, 2004.

12. T. Floquet and J.P. Barbot. A sliding mode approach for unknown input observers for linear systems. In Proceedings the IEEE Conference on Decision and Control, Bahamas, 2004.

13. T. Floquet and J.P. Barbot. An observability form for linear systems with unknown inputs. International Journal of Control, 79:132-139, 2006.

14. L. Fridman, J. Davila, and A. Levant. High-order sliding-mode observation and fault detection. Proceedings of the IEEE Conference on Decision and Control, pages 4317-4322, 2007.

15. L. Fridman, Y.B. Shtessel, C. Edwards, and X.G. Yan. Higher order sliding mode observer for state estimation and input reconstruction in nonlinear systems. International Journal of Robust and Nonlinear Control, 18:399-412, 2007.

16. Y. Hong, G. Chen, and L. Bushnell. Distributed observers design for leader-following control of multi-agent networks. Automatica, 44:846-850, 2008.

17. Y. Hu, J. Lam, and J. Liang. Consensus of multi-agent systems with Luenberger observers. Journal of the Franklin Institute, 350:2769-2790, 2013.

18. X. Ji and G. Chen. On the v-stability of complex dynamical networks. Automatica, 43:1049-1057, 2007.

19. C. Kambhampati, C. Perkgoz, R. J. Patton, and W. Ahamed. An interconnection predictive approach to faulttolerant control in network control systems. Journal of Systems and Control Engineering, 221:885-894, 2007.

20. J. Kautsky, N. K. Nichols, and P. Van Dooren. Robust pole assignment in linear state feedback. International Journal of Control, 41:1129-1155, 1985.

21. H.K. Khalil. Nonlinear Systems. Prentice Hall, Englewood Cliffs NJ., 1992.

22. A.J. Koshkouei and A.S.I. Zinober. Sliding mode controller-observer design for multivariable linear systems with unmatched uncertainty. Kybernetika, 36:95-115, 2000.

23. L.Gao, Y. Cui, X. Xu, and Y. Zhao. Distributed consensus protocol for leader-following multi-agent systems with functional observers. Journal of the Franklin Institute, 2015.

24. P. P. Menon and C. Edwards. Robust fault estimation using relative information in linear multi-agent networks. IEEE Trans. Autom. Control, 59:477 - 482, 2014.

25. P. P. Menon, C. Edwards, and N. Gomes Da Silva. An observer based controller design with disturbance feedforward framework for formation control of satellites. IET Control Theory and Applications, 2015.

26. P.P. Menon and C. Edwards. Decentralised static output feedback stabilisation and synchronisation of networks. Automatica, 45:2910-2916, 2009.

27. M. Mesbahi and M. Egerstedt. Graph Theoretic Methods in Multiagent Networks. Princeton Series in Applied Mathematics, 2010.

28. N. Meskin and K. Khorasani. Actuator fault detection and isolation for a network of unmanned vehicles. IEEE Trans. Autom. Control, 54:835-840, 2009.

29. R. J. Patton, C. Kambhampati, A. Casavola, P. Zhang, S. Ding, and A. D. Sauter. Generic strategy for fault-tolerance in control systems distributed over a network. European Journal of Control, 13:280-296, 2007.

30. F Plestan, Y Shtessel, V Bregeault, and A Poznyak. New methodologies for adaptive sliding mode control. International Journal of Control, 83:1907-1919, 2010.

31. R. Raoufi and A.S.I Zinober. Adaptive sliding mode observers in uncertain chaotic cryptosystems with a relaxed matching condition. International Workshop on Variable Structure Systems, pages 220-225, 2006.

32. G. Royle and C. Godsil. Algebraic graph theory. Springer Verlag, New York., 2001.

33. I. Shames, A. M. Teixeira, H. Sandberg, and K. H. Johansson. Distributed fault detection for interconnected secondorder systems. Automatica, 47:2757-2764, 2011. 
34. J. A. K. Suykens, P. F. Curran, and L. O. Chua. Robust synthesis for master slave synchronization of lure systems. IEEE Transactions on circuits and systems-I: Fundamental theory and applications, 46:841-850, 1999.

35. C. P. Tan and C. Edwards. Sliding Mode Observers for Robust Detection and Reconstruction of Actuator and Sensor Faults. International Journal of Robust and Nonlinear Control, 13:443-463, 2003.

36. C.P. Tan and C. Edwards. An LMI Approach for Designing Sliding Mode Observers. International Journal of Control, 74:1559-1568, 2001.

37. V.I. Utkin. Sliding Modes in Control Optimization. Springer-Verlag, Berlin, 1992.

38. B. L. Walcott and S. H. Żak. State Observation of Nonlinear Uncertain Dynamical Systems. IEEE Transaction on Automatic Control, 32:166-170, 1987.

39. Chi Wa Wu. Synchronisation of complex networks of nonlinear dynamical systems. World scientific publishing company, 2007.

40. Q. Wu and M. Saif. Robust fault detection and diagnosis for a multiple satellite formation flying system using second order sliding mode and wavelet networks. Proceedings of American Control Conference, 2007.

41. J. J. Yan, M. Hung, T. Y. Chiang, and Y. Yang. Robust synchronization of chaotic systems via adaptive sliding mode control. Physica A, 356:220-225, 2006.

42. X.G. Yan and C. Edwards. Nonlinear robust fault reconstruction and estimation using a sliding mode observer. Automatica, 43:1605-1614, 2007.

43. H. Zhang, F. Lewis, and A. Das. Optimal design for synchronization of cooperative systems: state feedback, observer, and output feedback. IEEE Transactions on Automatic Control, 56:1948-1952, 2011. 